\title{
Hallazgos y reflexiones en torno a la organización comunitaria: caso Nashira*
}

\section{Findings and Reflections on community organization: case Nashira}

\section{Descobertas e reflexões em torno à organização comunitária: caso Nashira}

Laura Victoria Cajiao Martínez**

Universidad de San Buenaventura, Cali, Colombia

Resumen. El artículo de investigación presenta los principales hallazgos encontrados en una organización comunitaria a partir de los elementos de la matriz comprensiva, instrumento de base para el análisis de organizaciones con características y/o prácticas innovadoras similares a las de la organización de estudio. Por medio de la matriz se identifica que Nashira ha estado orientada hacia el desarrollo de la mujer desde diferentes perspectivas, tratando de generar una democracia participativa, en donde la vida de cada una de sus participantes cobre otro sentido a

* $\quad$ Artículo de investigación, producto del proyecto: Organizaciones y Micropoderes Re-existentes, adscrito a la línea de investigación «Alta Dirección, Humanidades y el Educar» de la Maestría en Alta Dirección de Servicios Educativos. Universidad de San Buenaventura, Cali, Colombia. Fecha de Inicio: Noviembre de 2009. Fecha de terminación: Agosto de 2011.

** Psicóloga, magister en Alta Dirección de Servicios Educativos. Docente de la Facultad de Psicología de la Universidad de San Buenaventura, Cali y consultora de Desarrollo Organizacional. Miembro del Grupo de Investigación Alta Dirección, Humanidades y el Educar de la Universidad de San Buenaventura, Cali. Colombia. Email: lvcajiao@ usbcali.edu.co 
partir del intercambio, la transformación del conflicto, la capacitación, la construcción colectiva y el trabajo en equipo en los núcleos productivos, espacios que son tomados para la formación, el crecimiento y el avance del proyecto como un modelo de ecoaldea auto sostenible.

Palabras clave. Organización comunitaria, matriz comprensiva, proceso organizativo, convivencia y conflicto, sostenibilidad, núcleos productivos (Tesauro Unesco).

Abstract. This reflection paper present the main findings in a community organization, based on the comprehensive matrix elements, basic instrument for the analysis of organizations with similar characteristics or practices. Can be identify through the comprehensive matrix that Nashira has been directed towards the development of women from different perspectives, trying to create a participatory democracy, where the sense of life in each of its participants has another meaning through exchange, the transformation of conflict, training and team working in the production centers, spaces that are taken for formation, growth and progress of the project as a self-sustaining model ecovillage (Thesaurus Unesco).

Keywords. Community organization, comprehensive matrix, organizational process, coexistence and conflict, sustainability, production centers.

Resumo. O artigo de investigação apresenta as principais descobertas encontradas em uma organização comunitária a partir dos elementos da matriz compreensiva, instrumento de base para a análise de organizações com características e/ou práticas inovadoras similares às da organização de estudo. Por meio da matriz identifica-se que Nashira esteve orientada para o desenvolvimento da mulher desde diferentes perspectivas, tratando de gerar uma democracia participativa, na qual a vida de cada um de seus participantes tem outro sentido a partir do intercâmbio, da transformação do conflito, da capacitação, da construção coletiva e do trabalho em equipe nos núcleos produtivos, espaços que são tomados para a formação, para o crescimento e o avanço do projeto como um modelo de eco aldeia autossustentável.

Palavras chave. Organização comunitária, matriz compreensiva, processo organizativo, convivência e conflito, sustentabilidade, núcleos produtivos (Tesaurus Unesco). 


\section{Introducción}

Comprender cómo a través de otras formas de organización social, es posible encontrar espacios de coexistencia humana desde los cuales se rastrean elementos de la organización formal tradicional, sus experiencias y la gestión de la misma, ha sido uno de los objetivos planteados desde el Proyecto de Investigación: Organizaciones y Micropoderes ReExistentes. Como resultado investigativo, se presenta en este artículo los principales hallazgos encontrados en Nashira ${ }^{1}$, desde la perspectiva analítica, interpretativa y crítica de la autora y que son desarrollados en Organizaciones Comunitarias como opción de micropoder: Caso Nashira ${ }^{2}$. Ante los retos que el mundo demanda, tanto en el ámbito de la generación de ingresos desde el hogar y el empoderamiento de las mujeres, surge Nashira con el propósito de mejorar la calidad de vida de sus asociadas y sus familias por medio de la implementación de prácticas productivas innovadoras que están orientadas a la sostenibilidad del proyecto.

El estudio sobre el desarrollo de la comunidad, a partir de programas nacionales, regionales y locales, ha adquirido bastante importancia en América Latina, debido a las condiciones de empobrecimiento y subdesarrollo de la región (Ander-Egg, 2003). Las organizaciones comunitarias se han convertido en una alternativa de solución que puede llegar a satisfacer las necesidades básicas del individuo.

La ONU, enfatiza el papel de la participación social como generadora de capital social y desarrollo comunitario, señalando su importancia entre las características sociopolíticas y culturales de los contextos que favorecen la integración comunitaria y el bienestar social. De esta forma se empiezan a consolidar proyectos organizativos con el objetivo no solo de brindar seguridad básica a los individuos, sino también de aportar en su formación personal e interacción con el mundo (Martínez, 2004).

El proyecto Nashira como organización comunitaria, se empieza a consolidar a través de prácticas cotidianas, interacciones entre sus participantes y múltiples relaciones que establece con su entorno, lo

1 Proyecto de ecoaldea auto sostenible ubicada en el Municipio de Palmira y propuesto por la Asociación Mujeres Cabeza de Familia (ASOMUCAF).

2 Trabajo de Grado presentado para optar al título de Magister en Alta Dirección de Servicios Educativos. Autoras: Laura Victoria Cajiao M y Patricia Gómez E.

ITINERARIO EdUCATIVO • ISSN OILI-Z753 • AÑo XXVII, N. ${ }^{\circ}$ GI • ENERO - JUNIO DE ZOI3 • P. I5I-I66 
que permite erigirse como una opción de vida para muchas familias pobres que están por fuera de los círculos de producción y consumo y brinda la posibilidad de ser considerado, una opción en el sistema capitalista.

Las organizaciones comunitarias tradicionalmente se han abocado a ser colectivos problemáticos de exclusión social, marginales y de subdesarrollo económico y cultural; en un primer momento puede que Nashira no escape a esta visión, sin embargo, el proyecto empieza a consolidarse como una opción de educación y formación social, política, económica, cultural, de emprendimiento, auto sostenibilidad, con reconocimiento local, nacional e internacional, sobre la base de un espacio democrático, que aspira a mejorar la calidad de vida de las participantes y sus familias, a reducir los desequilibrios sociales, a suplir las necesidades básicas de alimentación, vivienda y educación.

\section{Metodología}

La metodología que orientó la investigación fue de tipo cualitativo, de carácter descriptivo, interpretativo y analítico. Durante el proyecto, para la comprensión de Nashira en su propia dinámica, se desarrolló la matriz ${ }^{3}$ que tiene como ámbito de estudio la organización, a partir de la mirada de diversos actores y permite considerar los diferentes aspectos estudiados en ésta:

Cuerpo de asociados (membresía)

Cuerpo directivo (en el nivel de las decisiones)

Cuerpo operativo (en el nivel de operar los «acuerdos», decisiones, la vida práctica y productiva de la organización, su convivencia, el conflicto y las relaciones internas)

Actores del entorno (la mirada desde afuera, la comunitaria, la externa)

3 Permite el abordaje de una serie de elementos y la interacción de los mismos con preguntas, generando un soporte empírico en la caracterización del proceso investigativo realizado. Elementos de análisis: proceso organizativo, proceso educativo, convivencia, crecimiento y subsistencia, género, vivienda y componente laboral. 
La figura 1 muestra la ruta metodológica por la que se transitó, facilitando la recolección, validación, análisis y comprensión de información:

ETAPAS METODOLÓGICAS

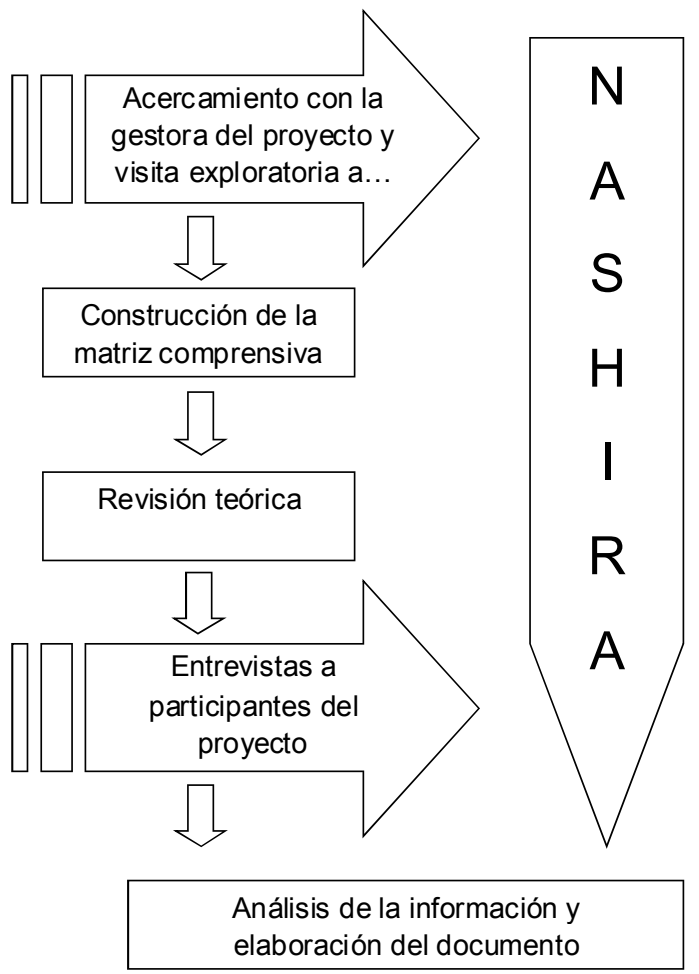

Figura 1. Ruta metodológica

Fuente: Proyecto de Investigación: Organizaciones y Micropoderes Re-existentes.

Los referentes teóricos permitieron reconocer aspectos de las organizaciones desde el pensamiento administrativo como: su lógica o dinámicas propias (Zapata, 2008), el lado humano de ellas (Cruz, Aktouf y Carvajal, 2003), su mirada a partir de lo público (Varela, 2005b) o tomando como referencia el Management (Varela, 2005a) y (Zapata, Murillo y Martínez, 2006). Desde lo comunitario, Ruíz (2004) y Fals Borda (1980) facilitan la comprensión de la organización comunitaria, la gestión asociada y la Investigación Acción Participativa (IAP), como método de investigación que pretende no sólo conocer las necesidades sociales de una comunidad, sino también agrupar esfuerzos para transformar la realidad con base en las necesidades sociales. 
Arias (2006) y Anand y Amartya (1996) con sus aportes, resaltan la importancia del Desarrollo Sostenible para el crecimiento y subsistencia de la organización y la comunidad; Freire (1985) y el Grupo de Investigación Interinstitucional: Historia de la práctica pedagógica en Colombia analizan como ha sido el proceso educativo en el país y la importancia de dichos procesos formativos en las organizaciones comunitarias como estrategia de empoderamiento para los asociados y la comunidad.

La Matriz Comprensiva, considerada por el equipo de investigadores como uno de los principales aportes desde el proyecto de investigación, para el análisis de organizaciones con características y/o prácticas innovadoras similares a las de Nashira como se mencionó anteriormente, se convierte a su vez en fundamento para el ejercicio de carácter reflexivo sobre los cuatro elementos considerados en el artículo, a partir de la posibilidad de que Nashira emerja como un modelo, el ser una organización de mujeres y su filosofía organizacional orientada a la sostenibilidad. De igual manera, este instrumento facilitó la correlación de elementos, es decir, al indagar sobre el proceso organizativo no se puede dejar de lado el componente laboral como característica particular en la división social del trabajo en núcleos productivos en esta organización comunitaria.

\section{Principales hallazgos}

A partir del trabajo de grado Organizaciones Comunitarias como opción de micropoder: Caso Nashira, se presentan a continuación los principales hallazgos y reflexiones realizadas sobre el proceso organizativo, la convivencia y el manejo del conflicto a través de la «Metáfora de las 88 Llaves», el crecimiento y sostenibilidad de la organización y el componente laboral en la organización comunitaria.

\section{Proceso organizativo}

Nashira se despliega con la idea de ser un modelo de desarrollo que genera ingresos para sus asociadas y de convertirse en un modelo de eco aldea auto sostenible en la región, en donde las mujeres se pueden transformar en motores de su propio desarrollo económico y a su vez, tengan herramientas para empoderarse.

La participación y asociación de actores externos en esta organización como el SENA, la Alcaldía de Palmira, la Embajada Británica, entre otros, 
permiten consolidar el concepto de Gestión Asociada al interior de este proyecto pues sin lugar a dudas, una de las herramientas más efectivas para empoderar a las asociadas, ha sido la capacitación y formación de ellas, objeto que han tenido los convenios establecidos con las organizaciones antes mencionadas. Tal como lo plantea Ruiz (2004): «la participación y capacitación de los más pobres en los programas y proyectos adquiere sentido si, al establecerse como parte de su gestión, contribuye a mejorar las condiciones de vida de la población» (p. 20). El proceso de gestión asociada en Nashira, la convierte en un proyecto de intervención local que coloca al Estado y a la organización comunitaria como protagonistas de los programas sociales que se desarrollan en el área.

Siendo considerada por su representante legal ${ }^{4}$ como una organización tradicional con elementos innovadores, está conformada la Junta Directiva de asociadas por una Presidente, una Vicepresidente, una Secretaria, una Tesorera, dos Vocales y el Representante Legal. Aunque se podría considerar de acuerdo a los aportes teóricos que se han hecho a la gestión de las organizaciones, que el proceso de toma de decisiones se encuentra centralizado en la cabeza de la junta directiva, se rastrea en las entrevistas y reuniones con los participantes, que las decisiones son tomadas por la ideóloga del proyecto.

A partir de este hallazgo, se hace importante identificar en Nashira el nivel de autonomía que tienen las asociadas respecto a la toma de decisiones y sobre el nivel de empoderamiento que poseen, entendiendo este proceso como «las acciones llevadas adelante para que quienes no tienen poder, se apropien de herramientas y estrategias que les permitan acumularlo» (Ruiz, 2004, p. 19). El empoderamiento no solo debe estar orientado a brindar herramientas formativas para la subsistencia y el crecimiento productivo, sino también hacia la autonomía en la toma de decisiones orientadas al desarrollo y sostenibilidad de la organización y para que tal como se plantea anteriormente, adquieran poder para ser motoras de su propio desarrollo.

Sus prácticas productivas caracterizadas por la división del trabajo en once núcleos productivos (cada uno conformado por ocho familias respectivamente) no solo son una muestra del trabajo en equipo sino que constituyen la coordinación y delegación de actividades,

4 Sr. Daniel Ruiz. Comunero y Juez de Paz de El Bolo La Italia, pertenece al proyecto desde el año 2003.

ITINERARIO EDUCATIVO • ISSN OILI-2753 • AÑO XXVII, N. ${ }^{\circ}$ GI • ENERO - JUNIO DE ZOI3 • P. I5I-I66 
responsabilidades y deberes de acuerdo a los comités como el de medio ambiente, recreación, mercadeo y relaciones públicas, así como las tareas, cuidados y mantenimiento que representa cada núcleo.

Nashira, como organización de mujeres cabeza de familia, debe fortalecer su capacidad de gestión, empoderar al cuerpo directivo buscando desarrollar habilidades técnicas en las asociadas y sus familias y fortalecer competencias en liderazgo, trabajo en equipo, toma de decisiones, diseño, ejecución y evaluación de proyectos así como el fortalecimiento de valores comunitarios, solidarios y participativos.

\title{
Convivencia y manejo del conflicto - metáfora de las 88 llaves
}

\author{
Viniendo de lejos, nos fuimos juntando. Sin pensarlo, \\ del campo resultaron las hojas \\ Y de uno en uno y de cinco en cinco y $d \epsilon$ ocho en ocho juntamos 88; \\ para $\in$ ntrar por $\in$ mismo \\ lugar donde entra una o la unión de los dos 8. \\ Mi libertad no termina donde inicia la de mi vecino, su casa ${ }^{5}$
}

La Metáfora de las 88 llaves es un ejercicio de carácter reflexivo que busca a través de dicho recurso literario, presentar las ideas, reflexiones y/o conceptos construidos a partir de los testimonios y experiencias de las asociadas y sus familias respecto a la convivencia y manejo del conflicto al interior de Nashira y lo que representa para ellas, tener su propia vivienda.

Hablar de convivencia en una comunidad donde habitarán 88 familias, cada una conformada en promedio por 3 habitantes, implica llegar al acuerdo de hacer 88 llaves para poder entrar por la misma puerta. Clavis ${ }^{6}$, raíz latina de la palabra llave, significa los clavos que usaron los antiguos romanos para cerrar sus puertas a los que daban diferentes formas para que cada dueño tuviera su propia «clave» y abrir la puerta de su propiedad. Por extensión, se utiliza la palabra metafóricamente para designar todo aquello a lo que es posible acceder por algún medio como la «llave del éxito», la «llave de la felicidad» o la «llave del paraíso»${ }^{7}$

5 Introducción a la metáfora construida por Harold Viafara S. Patricia Gómez E. Laura V. Cajiao M. Grupo de Investigación Alta Dirección, Humanidades y el Educar, Universidad de San Buenaventura Cali.

6 http://deconceptos.com/ciencias-sociales/llave. Consultada el 22 de Junio de 2011

7 http://deconceptos.com/ciencias-sociales/llave. Consultada el 22 de Junio de 2011 
Con los cimientos levantados por sus asociadas y sus familias, Nashira es vista como la oportunidad para acceder a un espacio propio, un espacio que no solo representa seguridad, bienestar y desarrollo a mediano y largo plazo, sino que representa también, el manejo del conflicto y la convivencia en un lugar en el que se trabaja y se habita a la vez. A modo de ejemplo se presenta este testimonio:

«Muy difícil de manejar la puerta, una puerta comunitaria muy complicada porque todos no tenemos el mismo sentido de cerrar, entonces la puerta mantiene muy abierta y pues siempre Nashira maneja muchas cosas al aire libre, entonces pues....muy complicada la puerta» (Entrevista a Bárbara Orjuela Presidenta de la Junta de Nashira y asociada del Proyecto. Marzo 5 de 2011).

El espacio residencial en donde se vive y aquellos conflictos que se presentan debido a la falta de tolerancia y respeto entre vecinos, la organización donde se trabaja y aquellos conflictos que se presentan también en relación a la toma de decisiones, el poder, los estilos de liderazgo, la falta de comunicación, falta de coordinación, entre otros, son un ejemplo de una realidad en Nashira. ¿Qué estrategias implementan para facilitar la convivencia al interior de la comunidad y manejar el conflicto? Respecto al tema Carmen Lozano, integrante de la comunidad apunta:

«En reuniones y todo eso, se discute y todo, con la junta, con la Doctora, ahí uno tiene que cambiar. Aquí hay unas normas, que no se pueden romper... tenemos, como esto va a ser un conjunto cerrado, hay una.... se me olvida como se llama eso...un manual de convivencia» (Entrevista a Carmen Lozano Vicepresidenta de la Junta de Nashira y Tesorera del núcleo productivo de codornices. Marzo 5 de 2011).

Por lo general, se tiende a asumir el conflicto como «situaciones negativas o no deseadas, lo que nos lleva a tratar de evitarlos, evadirlos, ocultarlos o eliminarlos. Sin embargo, los conflictos son situaciones de la vida cotidiana, que pueden ocurrir en el ámbito personal, el familiar, comunal, municipal, nacional e incluso internacional» (Cuadra, 2003, p. 15). Así mismo, se encuentra asociado a la confrontación de valores, estatus, poder, recursos escasos, entre otros. Algunas de las razones por las cuales se presentan conflictos en Nashira son las asignaciones de asociados a núcleos productivos que no son de su interés, no estar capacitado para estos y problemas de convivencia como la música a alto volumen hasta pasada la media noche, entre otros. He aquí otro testimonio: 
«Aquí no se puede colocar la música a alto volumen, llegar borrachito, todo eso, no se puede hacer escándalo ni nada, no se puede tener ni animalitos, perritos, pero yo tengo, yo las rompí» (Entrevista a Carmen Lozano, Vicepresidenta de la Junta de Nashira y Tesorera del núcleo productivo de codornices. Marzo 5 de 2011).

Una de las estrategias utilizada para la resolución de conflictos, es la intervención de un tercer actor no involucrado directamente en el conflicto, o lo que se conoce como mediación. Desplegar mecanismos de negociación, fortalecer la capacidad de negociación y habilidades de mediación en las asociadas y sus familias, son estrategias que soportadas en el Manual de Convivencia, regulan y facilitan la convivencia en la ciudadela ecológica, el componente laboral y el comportamental de los involucrados, orienta los procesos de convivencia de la comunidad y permiten vivir, por decirlo así, como es debido, fortaleciendo valores democráticos y participativos.

Frente a la pregunta de cómo perciben la convivencia y el manejo del manual al interior de la comunidad, manifiestan ser respetadas las normas establecidas en dicho manual, así lo muestra el siguiente testimonio:

«Si, la gente aquí sabe que hay cosas que no se deben de hacer y la gente no las hace y el que las hace ahí mismito se les llama la atención, entonces la gente para» (Entrevista a Margarita Mina, Asociada del Proyecto. Marzo 5 de 2011).

Cuando se violenta una llave, ésta tiende a quebrarse. Abusar de lo que constituye la llave, (acceso a mi espacio, acceso a mi libertad) es entrar en conflicto con el otro, es asumir que mi libertad no termina donde inicia la de mi vecino, es decir, su casa, recordando a la vieja y conocida frase: «mi libertad termina donde comienza la de mi vecino»; es pensar que mis acciones no tienen consecuencias o no afectan de alguna manera al otro.

Finalmente, las llaves también son un «instrumento que sirve para mantener cerrado y alejado de interferencias extrañas a cualquier cosa que se pretenda resguardar» ${ }^{8}$ Como cabezas de hogar, las asociadas de

8 http://deconceptos.com/ciencias-sociales/llave. Consultada el 13 de junio de 2011. 
Nashira son convocadas a resguardar a sus familias de la falta de acceso a la educación, a la salud y al trabajo en el país. Empoderadas estas mujeres y con el fin de alejar a sus familias del conflicto que representan las condiciones actuales, depositan su confianza en aquella «llave», aquel rayo de esperanza para tener un espacio propio en el que puedan subsistir y protegerse.

\section{Crecimiento y sostenibilidad de la organización}

Uno de los elementos claves para el desarrollo de Nashira, es la auto sostenibilidad, elemento que es considerado como uno de los principales objetivos de cualquier tipo de organización con fin de lucro, así como lo es la rentabilidad.

Esta organización posee un elemento innovador representado y concentrado en sus mujeres a quienes se orientan las herramientas y estrategias que las empodera, les permiten la ejecución de sus prácticas productivas auto sostenibles y las reúne en una opción de micropoder.

Si bien es cierto, Nashira como propuesta y tal vez, hasta como «modelo» sugiere o da cuenta de algunos elementos que le dan una condición sui géneris:

- Perspectiva medio ambiental

- Garantía de un territorio

- Garantía de vivienda

- Esquema productivo (núcleos productivos)

- Capacitación

La información recogida durante el proyecto de investigación, permite que emerja una pregunta de fondo iLa dinámica económica planteada en la propuesta Nashira garantiza la sostenibilidad a mediano y largo plazo? Se asume que para la etapa de desarrollo en la que se ingresó a Nashira, la organización contaba con estudios de viabilidad y/o factibilidad, estudios que se convierten en la estrategia que le permite a la organización garantizar su subsistencia, crecimiento y sostenibilidad así como analizar su viabilidad como proyecto. 
Sin embargo, tal como lo comenta Daniel Ruiz:

«La mayoría de los participantes de Nashira tienen otros ingresos económicos, puesto que aun la propuesta no es autosuficiente» (Entrevista a Daniel Ruiz, Representante Legal. Agosto 5 de 2010).

Por otro lado, resulta importante tener en cuenta como en este tipo de experiencias los principios filosóficos de la organización, pueden llegar a estar distantes con la realidad misma. En este sentido tendríamos que advertir cómo el deber ser, supera las condiciones reales de lo que pueden llegar a lograr las asociadas y sus familias, en tanto la dificultad de prever y proyectar, puede encontrar límites dadas las carencias de la existencia de un sistema o una línea de propuesta de coordenadas económicas.

Dos factores adicionales que se convierten en estrategias de crecimiento y subsistencia para la organización son: el Ecoturismo (el cual se retoma en el apartado de vivienda) y el Mercado Verde.

Un aspecto importante a considerar en cuanto al fomento del ecoturismo en Nashira, es la Política para el Desarrollo del Ecoturismo ${ }^{9}$ que se formula a nivel nacional y regional y establece los lineamientos preliminares para el desarrollo regional y local e identifica unas áreas temáticas prioritarias aplicables en el país. Su importancia radica en que si la organización plantea dentro de sus objetivos el ecoturismo, deberá acogerse a dichos lineamientos y cumplir con los requisitos exigidos para su fomento. Alojar visitantes extranjeros no necesariamente implica ecoturismo y se debe tener claridad frente a lo que se ofrece y la realidad del servicio.

El mercado verde como estrategia de subsistencia debe ir de la mano con el estudio de mercado que anteriormente se menciona, lo que le permite a la organización el análisis de la demanda, el posicionamiento de sus productos y sostenibilidad en el medio.

\section{Componente laboral - núcleos productivos}

Tal como se ha mencionado anteriormente, las asociadas se han organizado en once núcleos productivos entendiendo éste, como el centro

9 Ministerio de Comercio, Industria y Turismo. Ministerio de Ambiente, Vivienda y Desarrollo Territorial.Política para el Desarrollo del Ecoturismo. República de 
que convoca a un colectivo de ocho mujeres y sus familias para desarrollar una propuesta laboral en la búsqueda de subsistencia y sostenibilidad. Se identifica claridad en las asociadas frente a su rol u ocupación dentro de la organización a pesar de no existir un manual formal de funciones que especifique las tareas asignadas a cada uno, a propósito de esto Bárbara Orjuela, Presidente de la Junta, afirma:

Las personas que estamos viviendo que somos veinte familias, estamos totalmente organizadas, tenemos un oficio asignado.... Entonces cuando una familia va entrando se le va acomodando un oficio, todos los pedazos de Nashira tienen un doliente, yo sé quién aquí no ha barrido, yo sé porque el baño amaneció sucio, todo eso lo sabemos (Entrevista a Bárbara Orjuela, Presidente de la Junta y asociada del Proyecto. Marzo 5 de 2011).

Los núcleos productivos en Nashira han actuado como espacios de crecimiento no formal donde las mujeres se forjan en ellos y contribuyen a su vez con el avance de la propuesta, Bárbara Orjuela continua diciendo.

«Con los núcleos productivos estamos ahorita en un proceso de reorganización, sobre todo con unos núcleos que no estaban funcionando muy bien, vino un señor que está enseñándonos contabilidad, está mostrándonos si es viable que sigamos en ese núcleo, si podemos cambiar, que tanto hay que invertir, por ejemplo, nosotros tenemos pollos, entonces debemos saber que tantos pollos hay que meter para poder 8 familias tener plata de allí; ellas tienen codornices, que tantas codornices tienen que trabajar, el señor está indicándonos los caminos a seguir, todo esto es un proceso, todos los núcleos no se han movido igual, nunca es tarde, estamos aprendiendo, aprendiendo de los errores» (Entrevista a Bárbara Orjuela, Presidente de la Junta y asociada del Proyecto. Marzo 5 de 2011).

De igual manera que en las organizaciones productivas tradicionales, la división del trabajo en núcleos productivos dan cuenta del cuidado y mantenimiento, responsabilidades y deberes asociados a éstos.

El carácter ambiental, supuesto bajo el cual se organizan las personas en esta propuesta, se visualiza en la filosofía organizacional como eje transversal a todos los componentes de la organización, especialmente al componente laboral hacia donde orientan las estrategias ambientales 
(manejo del suelo, manejo del agua, tratamiento de basuras, entre otros) por medio del cual garantizan su sostenibilidad.

\section{A modo de conclusión}

Nashira se desarrolla como un espacio de coexistencia humana en donde se estimula la participación y los valores comunitarios. Sin embargo, conviene fortalecer la participación de las asociadas en cuanto a la dirección y desarrollo de la comunidad, es decir, brindarles herramientas para empoderarlas y darles autonomía en la gestión de la organización, la toma de decisiones, la negociación y el liderazgo del proyecto. Esto implica que se deben capacitar en técnicas productivas y agrícolas, establecer un proceso de formación en habilidades para la gestión financiera, administrativa, comercial y de los recursos, tanto materiales, como humanos, técnicas de negociación y manejo del conflicto, comunicación asertiva, liderazgo y planeación.

Al plantearse dentro sus objetivos el ser un «Modelo de Ecoaldea Sostenible», se compromete a cubrir y satisfacer en su totalidad los aspectos de estudios (formación), trabajo, ocio, necesidades diarias en las asociadas y sus familias. Así mismo, comprende que el enfoque sostenible, implica una vida que respeta y cuida el entorno, que practica una actividad agrícola tradicional, que utiliza construcciones bioclimáticas, recicla residuos, y aprovecha las energías renovables, entre otros, y que es más que tener simplemente un espacio en donde vivir. Teniendo en cuenta estas características y prácticas al interior de las ecoaldeas, la organización debe diseñar y poner en práctica estrategias como, el tratamiento de aguas residuales, ya que es una preocupación latente en la población; manejo de las basuras, conservación y preservación de los recursos naturales, entre otros, generando un proceso de transformación cultural y de filosofía organizacional asimilado y comprendido por parte de todas las asociadas y sus familias.

La convivencia, la armonía y el adecuado manejo del conflicto al interior de Nashira se convierten en un aspecto clave para el desarrollo de la organización pues el mal manejo de éstos, se puede convertir en un impedimento para el trabajo en equipo y la cooperación, fundamentales para el trabajo en los núcleos productivos, sabiendo que uno de los mayores problemas que se presentan en todas las organizaciones es el reunir a un grupo de personas para el cumplimiento de los objetivos organizacionales, 
ya que todas las personas tienen necesidades, intereses, conocimientos, experiencias, expectativas y otra serie de motivaciones diferentes para pertenecer a la organización, esto hace que los problemas de comunicación que se presentan al interior de los grupos, se deban generalmente a la personalidad y emotividad de cada uno de sus integrantes. Estas dificultades individuales y grupales deben ser identificadas por las líderes de Nashira y las coordinadoras de los núcleos y ser analizadas por todos los integrantes oportunamente con el fin de proponer y poner en marcha, estrategias y políticas que faciliten la convivencia y la promoción de los valores comunitarios al interior de la organización.

Nashira se convierte en un proyecto de intervención local que coloca al Estado y a la organización comunitaria como protagonistas de los programas sociales que se desarrollan en el área. Así mismo, permite identificar diferentes tipos de relaciones sociales que surgen al interior de la comunidad y el grado de complejidad en la división social del trabajo en ésta.

El proyecto busca la promoción del desarrollo de la mujer desde varias perspectivas, pasando de tener a las mujeres en una condición de subordinación y exclusión (histórica, cultural, política), a la construcción de un proyecto propio de vida que las coloca en un papel protagónico en el proceso de cambio y mejora de su realidad social; por lo tanto, el proyecto debe preservar la esencia del mismo, empoderando y potencializando a las mujeres en la resolución de conflictos, antecediéndose a las tensiones que generan la convivencia y el trabajo bajo un mismo techo.

\section{Referencias}

Anand, S. \& Amartya, K. S. (1996). Sustainable Human Development: Concepts and Priorities, United Nations Development Programme, Office of Development Studies Discussion Paper Series.

Ander-Egg, E. (2003). Repensando la investigación-acción-participativa. Argentina: Lumen, Humanitas.

Arias, F. (2006). Desarrollo sostenible y sus indicadores. En publicación: Documento de Trabajo no. 93. CIDSE, Centro de Investigaciones y Documentación Socioeconómica, Facultad de Ciencias Sociales, Universidad del Valle, Cali: Colombia. Diciembre. http:// bibliotecavirtual.clacso.org.ar/ar/libros/colombia/cidse/Doc93.pdf 
Cuadra, E. (2003). Cambio social y conflicto: Actores y Relaciones de poder/ Elvira Cuadra Lira; il. Kathy Sevilla Zelaya. - 1ed. - Managua: SIMAS

Cruz, F. Aktouf, O. \& Carvajal, R. (2003). El lado humano de las organizaciones. Cali: Universidad del Valle.

Fals Borda, O. (1980) La ciencia y el pueblo: nuevas reflexiones. En Salazar, María Cristina (editora) (1992). La investigación-acción participativa. Inicios y desarrollo. Consejo de Educación de Adultos de América Latina. Universidad Nacional de Colombia. Madrid: Editorial Popular, OEI, Quinto Centenario.

Freire, P. (1985). La educación como práctica de la libertad. México: Siglo Veintiuno Editores.

Martínez, M. (2004). Coaliciones Comunitarias: una Estrategia Participativa para el Cambio Social. En Intervención Psicosocial. Vol. 12. Num. 3. Pp. 251-267

Ruiz, V. (2004). Organizaciones comunitarias y gestión asociada. Una estrategia para el desarrollo de ciudadanía emancipada, 1era ed. Buenos Aires: Paidos.

Varela, E. (2005a). Dimensiones actuales de lo público a propósito de las interrelaciones entre Estado, Management Sociedad. Revista Pensamiento \& Gestión, (p. 38 - 68)

Varela, E. (2005b). La mercantilización de lo público. Cali: Universidad del Valle.

Zapata, A., Murillo, G \& Martínez, J. (2006). Organización y Management. Naturaleza, objeto, método, investigación y enseñanza. Cali: Universidad del Valle.

Zapata, A. (2008). Análisis y diseño organizacional. De la estructura funcional a la organización vacía. Cali: Universidad del Valle. 